\title{
Pericarditis aguda complicada con derrame pericárdico. Caso clínico pediátrico
}

\author{
Acute pericarditis, complicated by pericardial effusion in a pediatric patient: \\ case report
}

\author{
Dr. Daniel Palanca Arias" , Dra. Elena G. Corella Aznarb, Dra. Ariadna Ayerza Casasc, \\ Dra. Alba Fernández Gómez ${ }^{b}$ Dra. Marta López Ramón ${ }^{c}$ y Dr. Lorenzo Jiménez Montañés ${ }^{c}$
}

\begin{abstract}
RESUMEN
La pericarditis aguda es la enfermedad más común del pericardio en la práctica clínica. Supone el $0,1 \%$ de todos los ingresos hospitalarios y hasta un $5 \%$ de aquellos por dolor torácico.

En países desarrollados, la causa suele ser benigna, y son más frecuentes las idiopáticas e infecciosas que las secundarias a pericardiotomía quirúrgica y neoplasias. La tuberculosis es la causa más importante en países en vías de desarrollo. Los síntomas más comunes son el dolor torácico característico y la fiebre. Debido a su benignidad y buena evolución con reposo y tratamiento médico, se puede controlar de forma ambulatoria, teniendo presentes aquellos signos de alarma para vigilar o pacientes de riesgo para evitar complicaciones (derrames importantes, taponamiento cardíaco, recurrencias, etc.). Presentamos un caso clínico de un niño de 7 años con antecedentes de comunicación interauricular cerrada quirúrgicamente 9 meses antes, con un cuadro de pericarditis aguda de evolución favorable.

Palabras clave: dolor torácico, pericarditis aguda, derrame pericárdico, electrocardiografía, síndrome pospericardiotomía.
\end{abstract}

\section{ABSTRACT}

Acute pericarditis is the most common disease of the pericardium encountered in clinical practice. It is diagnosed in $0.1 \%$ of all admissions and $5 \%$ of emergency room admissions for chest pain.

In developed countries, it is usually due to a benign cause. Idiopathic and infectious pericarditis are more common than secondary to surgical pericardiotomy or neoplastic causes, whereas tuberculosis is the dominant cause in developing

a. Hospital Infantil Miguel Servet, Zaragoza, Unidad de Cardiología Pediátrica, Unidad de Cuidados Intensivos Pediátricos.

b. Hospital Infantil Miguel Servet, Zaragoza, Unidad de Pediatría.

c. Hospital Infantil Miguel Servet, Zaragoza, Unidad de Cardiología Pediátrica.

Correspondencia:

Dr. Daniel Palanca Arias: danielpalanca@hotmail.com

Financiamiento: Ninguno.

Conflicto de intereses: Ninguno que declarar.

Recibido: 14-11-2016

Aceptado: 15-2-2017 countries. The most common symptoms of pericarditis are characteristic chest pain and fever. Since pericarditis presents a benign outcome because of self-limiting and good response to conventional anti-inflammatory therapy, it can be safely managed on outpatient basis unless a specific cause is suspected or the patient has high-risk features to avoid complications such as pericardial effusion, cardiac tamponade or recurrent pericarditis. We report a case of pericarditis, diagnosed 9 months after surgical closure of an atrial septal defect, in a 7-year-old boy with favorable evolution.

Key words: chest pain, acute pericarditis, pericardial effusion, electrocardiography, postpericardiotomy syndrome.

http: / / dx.doi.org/10.5546/aap.2017.e237

Cómo citar: Palanca Arias D, Corella Aznar EG, Ayerza Casas A, et al. Pericarditis aguda complicada con derrame pericárdico. Caso clínico pediátrico. Arch Argent Pediatr 2017;115(4):e237-e242.

\section{INTRODUCCIÓN}

El dolor torácico es un motivo de consulta relativamente frecuente en los servicios de urgencias de pediatría. Las causas idiopáticas y musculoesqueléticas son las más frecuentes; un pequeño porcentaje son de origen cardíaco, entre las que la pericarditis aguda es la causa más usual. En aquellos pacientes operados de cardiopatías congénitas, se deberá tener en cuenta el síndrome pospericardiotomía, tras la exclusión de otras causas posibles de pericarditis.

La pericarditis suele presentar una etiología benigna y autolimitada, y aparecen, ocasionalmente, complicaciones durante su evolución. Por ello, es importante conocer el rendimiento de las pruebas complementarias y sus cambios evolutivos, que aportan información diagnóstica y terapéutica, así como informar de los síntomas de alarma para vigilar en el domicilio.

\section{CASO CLÍNICO}

Niño de 7 años que acude al Servicio de Urgencias por dolor torácico de unas horas de evolución que mejora al inclinarse hacia delante y empeora en decúbito prono. Afebril. Sin 
antecedente infeccioso ni traumatismo torácico. Intervenido por comunicación interauricular tipo ostium secundum (CIA-OS) amplia, corregida quirúrgicamente hace 9 meses sin incidencias posoperatorias ni en los controles posteriores. Bien vacunado.

Se realiza una radiografía de tórax (Figura 1. a) y un electrocardiograma (Figura 2. a). Dados los antecedentes del paciente, es visto por Cardiología Infantil y se realiza una ecocardiografía transtorácica (ETT) (Figura 3). Ante los hallazgos en el electrocardiograma y la sospecha clínica de pericarditis aguda, se recomienda reposo y tratamiento con antiinflamatorios no esteroideos (AINE). Dada la ausencia de factores de riesgo, se indican los signos de alarma para vigilar en el domicilio y el control posterior en las consultas. A las 24 horas, inicia un cuadro de fiebre acompañado de malestar general, sudoración y aumento de dolor irradiado a la extremidad superior izquierda y el cuello, que se acentúa con la inspiración. Es reevaluado en Urgencias y se destaca, en la exploración física, palidez y sudoración con adecuada perfusión periférica y pulsos distales presentes. Las cifras de tensión arterial y de frecuencia cardíaca (FC) son normales y no se aprecia pulso paradójico ni roce pericárdico en la auscultación cardíaca. Ante el empeoramiento clínico, se realiza una nueva radiografía de tórax (Figura 1. b), sugestiva de aparición de derrame pericárdico, confirmado en la ETT (Figuras 4. a y 4.b), y un electrocardiograma de control (Figura 2. b). Ante la rapidez de la instauración del derrame pericárdico moderado (11-12 mm) y el brusco empeoramiento clínico, se decide la monitorización y la vigilancia estrecha en la Unidad de Cuidados Intensivos Pediátricos (UCIP).

Durante su ingreso, se amplía la batería de estudios para despistaje infeccioso y otras posibles causas, y se destaca, únicamente, una discreta leucocitosis con neutrofilia, que se resuelve en la analítica de control a las $48 \mathrm{~h}$. Fracción $\mathrm{N}$-terminal del péptido natriurético cerebral (ProBNP): 588,2 $\mathrm{pg} / \mathrm{mL}(0-300 \mathrm{pg} / \mathrm{mL})$; troponina $\mathrm{I}:<0,01 \mathrm{ng} / \mathrm{mL}$ (0-0,04 $\mathrm{ng} / \mathrm{mL})$; creatina quinasa (CK): $77 \mathrm{U} / \mathrm{L}$ (0-171 U/L); proteína C reactiva (PCR): 7,42 mg/ $\mathrm{dL}(0-0,5 \mathrm{mg} / \mathrm{dL})$; y procalcitonina: $0,55 \mathrm{ng} /$ $\mathrm{mL}(0-0,5 \mathrm{ng} / \mathrm{mL})$. Función renal, hepática y tiroidea: normal. Las serologías extraídas para enfermedad de Lyme, virus de Epstein-Barr, parvovirus B19, micoplasma, citomegalovirus, rubéola, toxoplasma y PCR para enterovirus fueron negativas, así como el hemocultivo, la prueba de la tuberculina y el exudado faríngeo de enterovirus mediante PCR.

Figura 1. Evolución de la radiografía de tórax. Figura 1. a. Radiografía de tórax al momento del diagnóstico (día 1): normal. Suturas de esternotomía media. Figura 1. b. Cardiomegalia (día 2). Con respecto al estudio previo, 24 horas antes, se muestra un aumento de la silueta cardiopericárdica con un pinzamiento del seno cardiofrénico, sugestiva de derrame pericárdico. Se aprecia una silueta cardíaca globular en forma de "cantimplora o botella de agua"
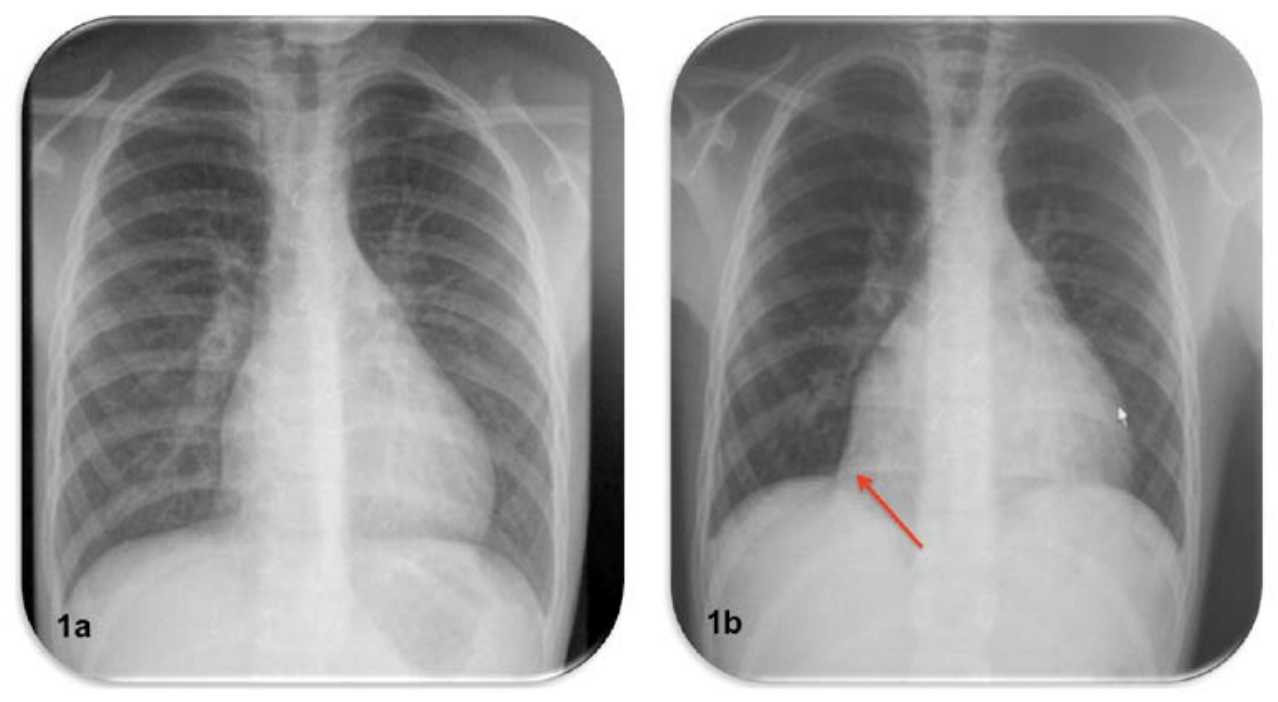
Ante el diagnóstico de exclusión definitivo de pericarditis aguda idiopática con derrame pericárdico, se inicia un tratamiento con aspirina (ácido acetilsalicílico -AAS-) (100 mg/ kg/ día cada $6 \mathrm{~h}$ ) y colchicina $(0,5 \mathrm{mg} /$ día), con buena evolución del dolor y mejoría del estado general. Por la estabilidad clínica, puede ser dado de alta a las $72 \mathrm{~h}$ del ingreso, y se recomienda reposo y mantener el tratamiento médico. La evolución es satisfactoria con desaparición de la clínica a la semana del inicio del tratamiento. Permanecen los signos electrocardiográficos de pericarditis en resolución (Figura 2. c) durante el primer mes con normalización completa del electrocardiograma

FIgURA 2. Fases en la evolución del electrocardiograma durante la enfermedad. 2. a (día 1). Electrocardiograma fase I: ritmo sinusal a 74 latidos por minuto (lpm), elevación del ST de $1 \mathrm{~mm}$ en derivaciones II, aVF, V2, con onda T positiva. Bloqueo incompleto de rama derecha. QRS estrechos. Eje $+60^{\circ}$. Repolarización: descenso de ST de $1 \mathrm{~mm}$ en V3. 2. $b$ (día 2). Electrocardiograma fase II: descenso del ST y aplanamiento de onda T. 2. c (1 mes). Electrocardiograma fase III: inversión de la onda T. 2. d (2 meses). Electrocardiograma fase IV: normalización
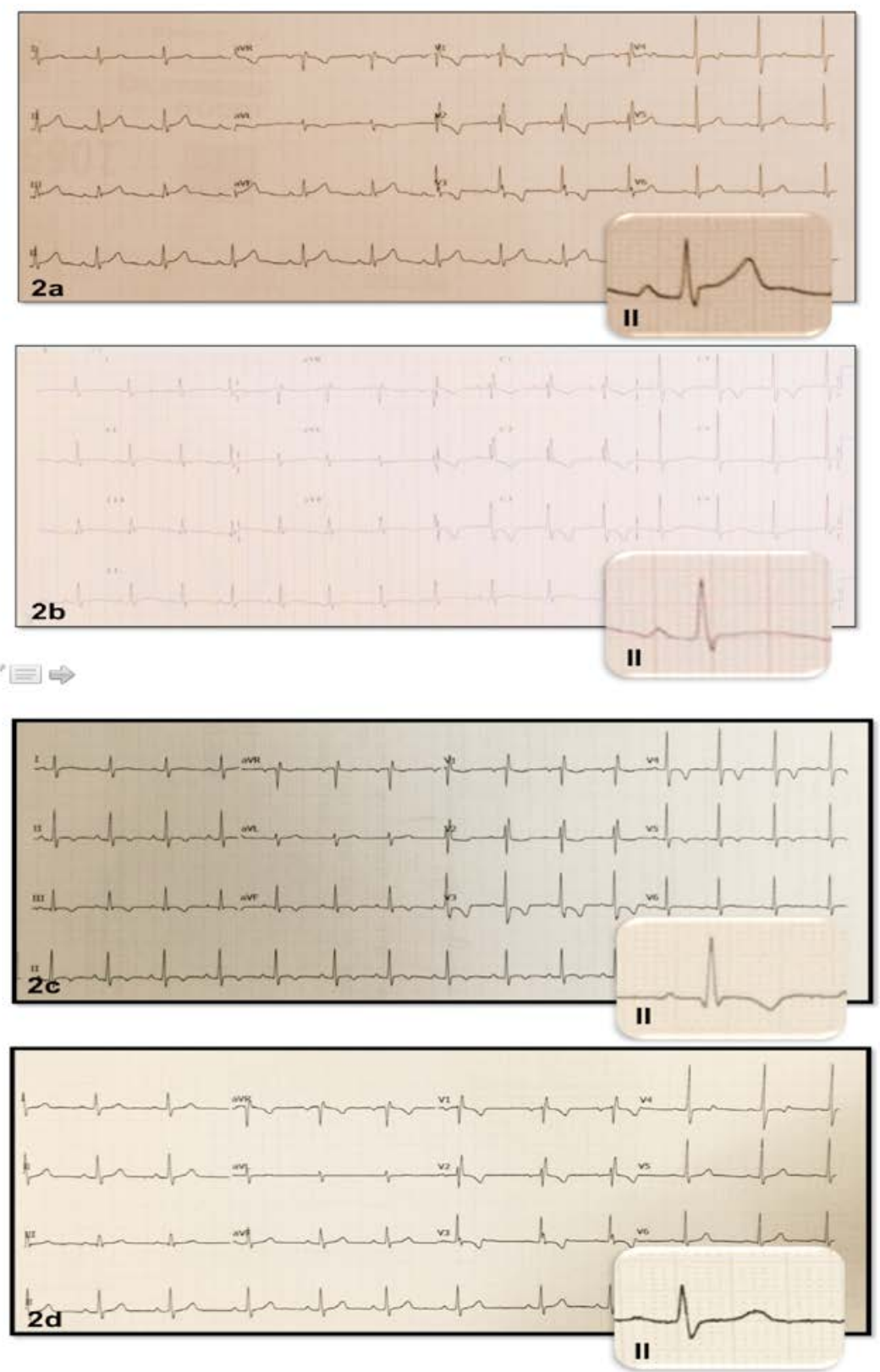
(Figura 2. d) a los 2 meses del cuadro. Al pasar un mes del evento, se recomienda reposo relativo y se retira la AAS; puede suspender la colchicina a los 3 meses, con adecuada respuesta, y reanuda la actividad física.

\section{DISCUSIÓN}

La pericarditis aguda es un síndrome clínico debido a una infiltración del pericardio por células inflamatorias. El hallazgo más característico es el dolor torácico punzante, por lo general, de localización retroesternal y precordial izquierda, con frecuencia, irradiado al cuello y al epigastrio o hacia ambos trapecios como consecuencia de la compresión del nervio frénico. Característicamente, el dolor aumenta en decúbito prono, con la tos, la inspiración profunda o la deglución y mejora al inclinarse hacia delante, debido a una disminución de la presión en el pericardio parietal. Como síntomas asociados, pueden aparecer también malestar, fiebre, tos o disnea. Un signo característico en la auscultación es el roce pericárdico, que se escucha mejor si el paciente se encuentra sentado y durante la inspiración, aunque su ausencia no excluye el diagnóstico. ${ }^{1,2,3}$ Las alteraciones electrocardiográficas seriadas son muy útiles para su diagnóstico. Los cambios en el electrocardiograma pueden ponerse de manifiesto ya a las pocas horas del inicio del dolor torácico. Se describen 4 estadios clínicos característicos ${ }^{4}$
(Figura 2), que solo ocurren de forma completa en la mitad de los pacientes.

El diagnóstico de pericarditis aguda se puede hacer cuando existen, al menos, dos de los tres criterios: dolor torácico característico, roce pericárdico o dichos cambios evolutivos de la repolarización ventricular a nivel del electrocardiograma. El roce pericárdico aislado en la auscultación es por sí mismo un hallazgo patognomónico. La pericarditis puede presentarse con derrame si el líquido pericárdico supera los 50 $\mathrm{ml}$ de volumen ${ }^{5} \mathrm{o}$ con taponamiento cardíaco ${ }^{6}$ si el derrame es importante o se instaura rápidamente.

Los objetivos del tratamiento son el alivio del dolor y la resolución de la inflamación y el derrame pericárdico si está presente. Deberá ir encaminado a tratar la causa y descartar aquellas que dispongan de tratamiento (bacteriana, tuberculosis, hongos, etc.). Inicialmente, se recomienda reposo en cama con la cabecera elevada $\left(45-60^{\circ}\right)$ y analgesia oral con AINE, como ibuprofeno (15-30 mg/ kg/ día en 3-4 dosis) o indometacina (1-3 mg/ kg/ día, máximo de 100 $\mathrm{mg}$ / día, en 3-4 dosis), durante unas 2-3 semanas sobre la base de la persistencia de los síntomas. Una combinación de uso habitual suelen ser los salicilatos (AAS: $100 \mathrm{mg} / \mathrm{kg} /$ día en 4 dosis) durante un mínimo de 2 semanas y colchicina (0,5 mg/ día) asociada unos 3 meses con retirada paulatina. ${ }^{7}$ El uso asociado de colchicina más AINE ayuda a reducir los síntomas, disminuir

FIGURA 3 (día 1). Ecocardiografía (eje paraesternal largo) normal, sin evidencia de derrame pericárdico

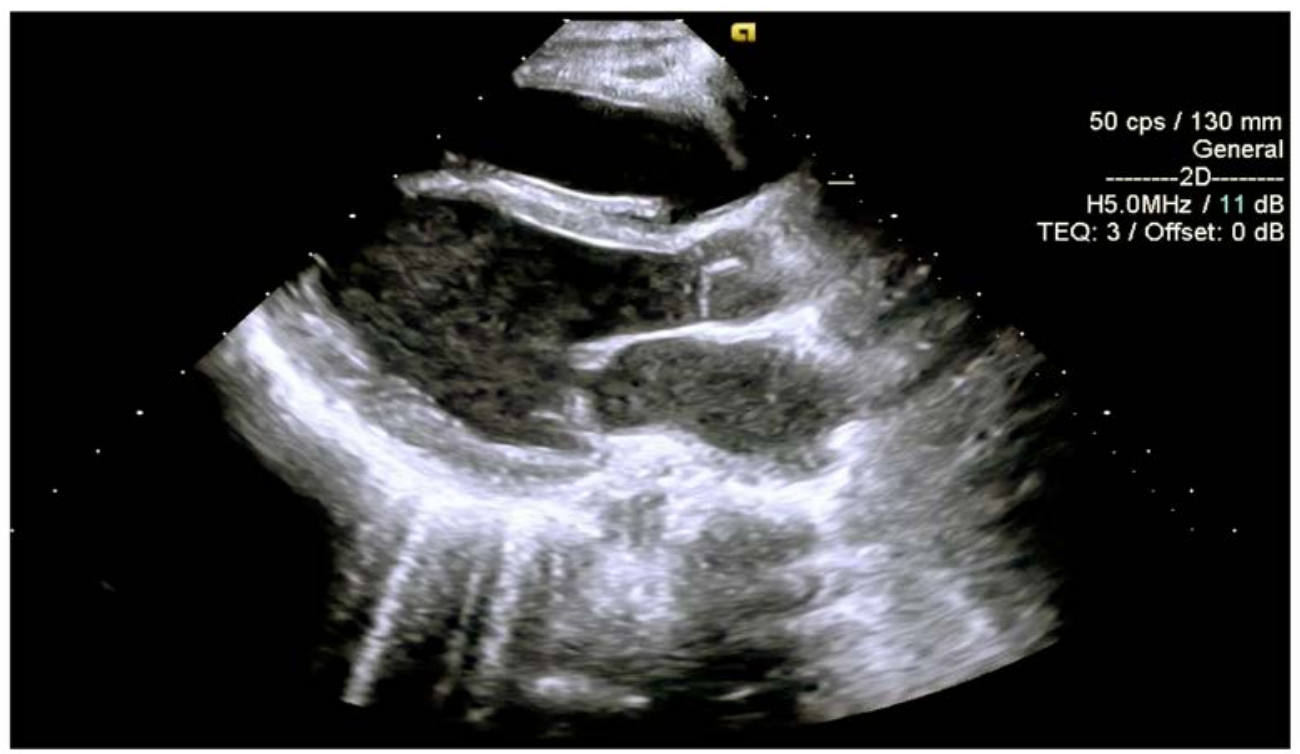


las recurrencias $\mathrm{y}$, generalmente, presenta buena tolerancia. ${ }^{5,8,9}$ Los corticoides (1-2 mg/ kg/ día), prednisona o similar, no se recomiendan como primera elección, solo en casos refractarios o aquellos en los que persiste dolor intenso o fiebre prolongada más de una semana, para pacientes con contraindicaciones y falta de respuesta a AAS o AINE, pericarditis urémica o secundaria a enfermedades del tejido conectivo. Solo en casos determinados, se recurre a la pericardiocentesis asociada a un derrame pericárdico que sugiera un origen purulento o si el taponamiento cardíaco es grave.

Como diagnóstico diferencial en un paciente sometido a una operación cardíaca, se deberá tener en cuenta el síndrome pospericardiotomía o síndrome de Dressler, que suele manifestarse al final de la primera semana del posoperatorio y puede aparecer, incluso, meses después. ${ }^{10}$ Se presenta con fiebre, malestar general, dolores articulares, irritabilidad, síntomas de inflamación pericárdica y pleural, y disminución del apetito. La etiología es desconocida, pero se postula que se trata de un proceso autoinmune desencadenado por la cirugía. Se presenta hasta en un $30 \%$ de aquellos pacientes con apertura pericárdica en la cirugía y, con mayor frecuencia, tras la cirugía de tetralogía de Fallot y en el cierre de CIA o comunicación interventricular (CIV). Aunque infrecuente, puede ser recurrente y se han descrito casos que desarrollaron pericarditis constrictiva. ${ }^{11}$ El diagnóstico es fundamentalmente clínico y ecocardiográfico. En la auscultación, es frecuente la presencia de roce pericárdico. En el electrocardiograma, no existen cambios específicos, a diferencia de los cambios evolutivos bien definidos en el caso de la pericarditis aguda.

Con el presente caso, se refleja la importancia de la evolución en el tiempo que describe los estadios del electrocardiograma típicos de la pericarditis y la progresión a través de la imagen (radiografía de tórax y ecocardiografía), que, en ocasiones, puede ser normal al inicio y restar importancia. Además, la vigilancia en la UCIP del derrame pericárdico fue prioritaria, no solo debido a la cantidad absoluta de líquido, sino por la rapidez con que se acumuló, los antecedentes quirúrgicos y las características del pericardio.

Figura 4 (día 2). Ecocardiografía (eje subcostal largo). b1: separación de ambas hojas del pericardio durante todo el ciclo cardíaco, con contenido anecoico en su interior, indicativo de derrame pericárdico moderado (11-12 mm en telediástole), loculado y con numerosos tractos de fibrina en su interior (flecha) sin signos de taponamiento; b2: ecocardiografía en modo M: medición de la cuantía de derrame.
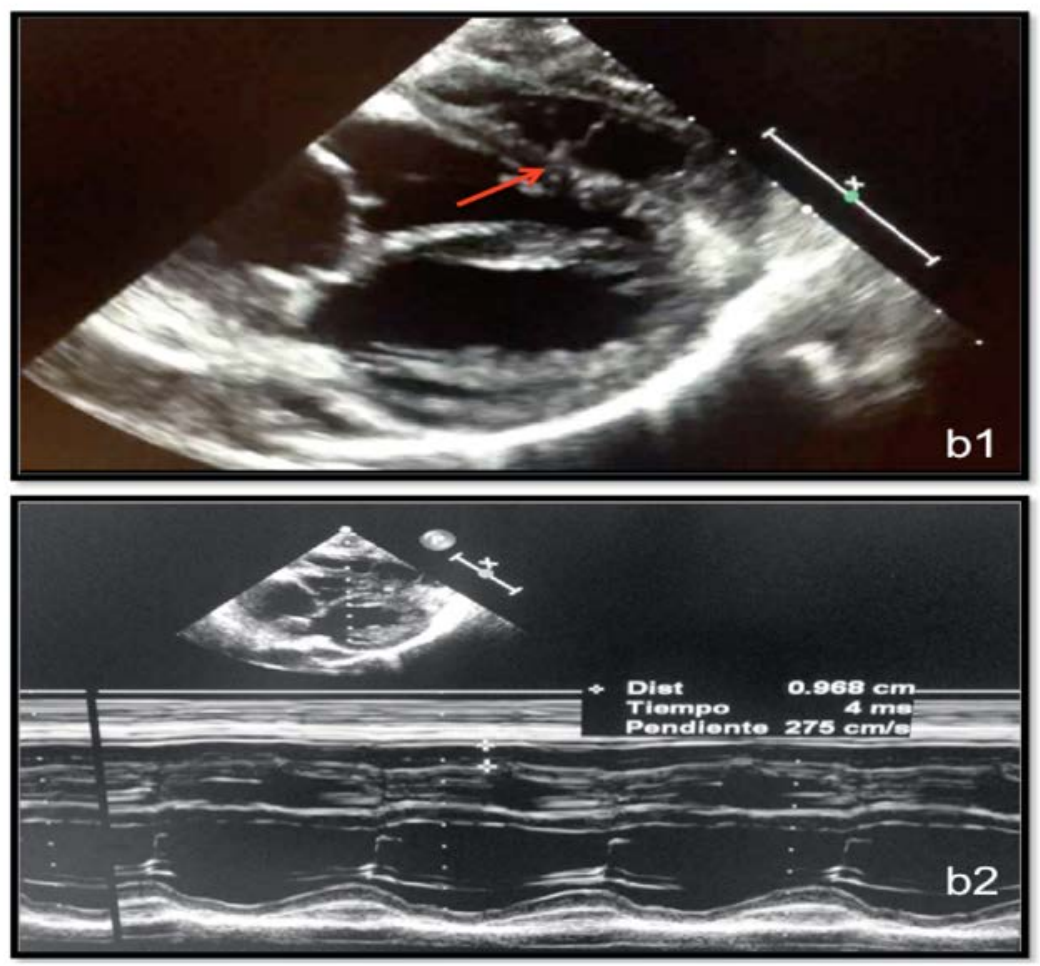
Debido al curso relativamente benigno de la pericarditis y que la gran mayoría de ocasiones son por causas comunes (idiopática, viral), no suele ser necesario buscar la etiología en todos los pacientes. ${ }^{12}$ Por ello, la mayoría de los pacientes son tratados por una teórica causa viral con AINE y colchicina. ${ }^{13,14} \mathrm{Si}$ el paciente no presenta factores de riesgo añadidos (analítica realizada con leucocitosis o troponinas cardíacas elevadas, ecocardiografía con derrame mayor de $20 \mathrm{~mm}$, estado de inmunodepresión, tratamiento con antagonistas de la vitamina $\mathrm{K}$, antecedente de traumatismo agudo), antes de dar el alta al domicilio, es importante tener presente el cumplimiento del las recomendaciones iniciales e informar de los signos de alarma que deben vigilar en su domicilio (fiebre $>38^{\circ} \mathrm{C}$, falta de respuesta tras una semana con AINE) para volver a consultar. ${ }^{13}$

\section{REFERENCIAS}

1. Longás Tejero MA, Sanz Barrio A, Luquero Bachiller F, et al. Pericarditis aguda. Medicine - Programa de Formación Médica Continuada Acreditado 2013;11(43):2541-58.

2. Crespo Marcos D, Pérez-Lescure Picarzo J, Zambrano Castaño M. Dolor torácico. Rev Pediatr Aten Primaria 2010;12(45):95-107.

3. FerrésiSerratF, García Algas F. Dolor torácico.En:Protocolos diagnóstico-terapéuticos de Urgencias Pediátricas SEUP-AEP. Asociación Española de Pediatría, Sociedad Española de Urgencias Pediátricas. 2. ${ }^{\mathrm{a}}$ ed. Madrid:Ergón; 2010.Págs.8390. [Acceso: 20 de agosto de 2016]. Disponible en: http:/ / www.aeped.es/sites/default/files/documentos/dolor_ toracico.pdf.
4. Park M. Electrocardiografía. En Park M. Cardiología Pediátrica. 6. ${ }^{\text {ta }}$ ed. Barcelona: Elsevier; 2015.Pág.63.

5. Adler Y, Charron P, Imazio M, et al. 2015 ESC Guidelines for the diagnosis and management of pericardial diseases: The Task Force for the Diagnosis and Management of Pericardial Diseases of the European Society of Cardiology (ESC) Endorsed by: The European Association for CardioThoracic Surgery (EACTS). Eur Heart J 2015;36(42):2921-64.

6. García Angleu F, González Vila L, Herrera del Rey C. Dolor torácico en el niño. En Protocolos sociedad Cardiología pediátrica y cardiopatías congénitas del niño y del adolescente. Barcelona: CTO; 2010.Pags.101-10. [Acceso: 1 de septiembre de 2016]. Disponibleen:http:/ / www.secardioped.org/modules.ph p? name $=$ webstructure\&lang $=$ ES\&idwebstructure $=21$.

7. Johnson Louis M, Vargas GonzálezS, Mendoza JaramilloO. Pericarditis: tratamiento cardíaco. En: Lopez-Herce Cid J, Calvo Rey C, Rey Galán C, etal.Manual decuidados intensivos pediátricos. 4. ${ }^{a}$ ed. Madrid: Publimed; 2013.Págs.266-70.

8. Imazio M. Contemporary management of pericardial diseases. Curr Opin Cardiol 2012;27(3):308-17.

8. Imazio M, Brucato A, Belli R, et al. Colchicine for the prevention of pericarditis: what we know and what we do not know in 2014 - systematic review and meta-analysis. J Cardiovasc Med (Hagerstown) 2014;15(12):840-6.

9. Pérez-Muñuzuri A, Fuster-Siebert M, Bravo-Mata M, et al. Síndrome pospericardiotomía: recurrencias tardías. An Esp Pediatr 2002;56(4):369-70.

10. Kim BJ, Ma JS. Constrictive pericarditis after surgical closure of atrial septal defect in a child. J Korean Med Sci 1998;13(6):658-61.

11. Gouriet F, Levy PY, Casalta J, et al. Etiology of Pericarditis in a Prospective Cohort of 1162 Cases. Am J Med 2015;128(7):784.e1-e8.

12. Imazio M. Treatment of acute pericarditis. In: UpToDate, Post TW (Ed), UpToDate, Waltham, MA. [Acceso: 10 de agosto de 2016]. Disponible en: http:/ /www.uptodate. $\mathrm{com} /$ contents / treatment-of-acute-pericarditis.

13. Raval J, Nagaraja V, Eslick GY, et al. The Role of Colchicine in Pericarditis -A Systematic Review and Meta-analysis of Randomised Trials. Heart Lung Circ 2015;24(7):660-6. 